\title{
Association between periostin and epithelial-mesenchymal transition in esophageal squamous cell carcinoma and its clinical significance
}

\author{
YA-JING LV ${ }^{1 *}$, WEI WANG ${ }^{1 *}$, CHU-SHU JI ${ }^{1}$, WEI JIA ${ }^{1}$, MING-RAN XIE $^{2}$ and BING HU ${ }^{1}$ \\ Departments of ${ }^{1}$ Medical Oncology and ${ }^{2}$ Thoracic Surgery, Anhui Provincial Hospital, \\ Anhui Medical University, Hefei, Anhui 230001, P.R. China
}

Received December 9, 2015; Accepted February 3, 2017

DOI: $10.3892 / \mathrm{ol} .2017 .6124$

\begin{abstract}
The present study aimed to investigate the association between periostin (POSTN), epithelial cadherin (E-cad) and vimentin (Vim) expression levels in esophageal squamous cell carcinoma (ESCC) tissues, and its clinicopathological significance. A total of 58 patients with esophageal cancer were enrolled. Immunohistochemistry was performed to quantify the expression levels of POSTN, E-cad and Vim. E-cad expression was reduced in ESCC tissue, which was associated with severe tumor node metastasis (TNM) stage $(\mathrm{P}<0.001)$, lymphatic metastasis $(\mathrm{P}<0.001)$ and vascular invasion $(\mathrm{P}=0.026)$. Conversely, Vim expression was found to be increased in ESCC tissues, and had associations with TNM stage $(\mathrm{P}=0.039)$ and lymphatic metastasis $(\mathrm{P}=0.039)$. POSTN overexpression observed in ESCC cells was associated with attenuation of $\mathrm{E}$-cad expression $(\mathrm{P}<0.001)$ and elevated expression levels of Vim $(\mathrm{P}<0.001)$. Additionally, significant correlations between the overexpression of POSTN in ESCC cells and clinicopathological variables including TNM staging $(\mathrm{P}=0.009)$, degree of differentiation $(\mathrm{P}<0.001)$, lymphatic metastasis $(\mathrm{P}=0.009)$ and vascular invasion $(\mathrm{P}=0.002)$ were verified. Multivariate analysis revealed that overexpression of POSTN in ESCC cancer cells is able to predict the poor prognosis of patients independently of overall survival $(\mathrm{P}=0.022)$ and disease free survival $(\mathrm{P}=0.019)$. The preliminary findings of the present study demonstrate that POSTN is involved in the epithelial-mesenchymal transition of ESCC cells, and may
\end{abstract}

Correspondence to: $\mathrm{Dr}$ Bing $\mathrm{Hu}$, Department of Medical Oncology, Anhui Provincial Hospital, Anhui Medical University, 17 Lujiang Road, Hefei, Anhui 230001, P.R. China

E-mail: hubing2013oncol@gmail.com

${ }^{*}$ Contributed equally

Key words: esophageal squamous cell carcinoma, periostin, epithelial cadherin, vimentin, epithelial-mesenchymal transition, clinicopathological factors therefore be a predictive factor for tumor invasion and metastasis, as well as an indicator of poor prognosis for patients with ESCC.

\section{Introduction}

Esophageal cancer is the fourth leading cause of cancer mortality worldwide, and is one of the most aggressive types of digestive tract tumor (1). The poor prognosis of esophageal cancer is due to the high incidence rate of lymph node metastasis and the invasion of neighboring organs within a 5-year period following surgery, despite improvements in treatment (2). Investigations into the mechanisms underlying invasion and metastasis in esophageal cancer may contribute to an improved patient prognosis.

Loss of epithelial-cadherin (E-cad) and simultaneous acquisition of vimentin (Vim) in epithelial cells is an alteration indicative of the epithelial-mesenchymal transition (EMT), which, in tumors, is a crucial step for cancer cells to obtain malignant properties (3). The epithelial transmembrane glycoprotein E-cad, a cell adhesion molecule, can regulate cell motility in a calcium-dependent manner (4). Dysregulated E-cad expression is able to induce impairments in adhesive ability and abnormalities in differentiation due to genetic mutation, promoter region hypermethylation and suppression of transcription, allowing tumor cells to dissociate from the primary lesion (5).

Transcriptional repression is considered one of the most critical mechanisms underlying the downregulation of E-cad expression. The type III intermediate filament protein Vim is normally expressed in cells of mesenchymal origin. Vim is a component of the cytoskeleton, is involved in cell adhesion and migration, and also regulates apoptosis and signal transduction in tumor tissues (6-8). EMT, as a target involved in invasion and metastasis mechanisms, has been revealed to result in the poor prognosis of patients with triple negative breast cancer and pancreatic cancer $(9,10)$. EMT may be directly or indirectly mediated by various factors including snail, slug and twist. Signaling of transforming growth factor- $\beta$ is considered to exert a pivotal function in triggering and promoting EMT (11). Periostin (POSTN) is an important downstream target in this process, and has been implicated to mediate the 
initiation of EMT triggered by twist in prostate cancer (11). As such, POSTN is considered as a key proteins involved in EMT.

POSTN, also termed osteoblast-specific factor 2, is an evolutionarily conserved extracellular matrix (ECM) protein and a member of the fasciclin family (12), and has been reported to be overexpressed in various types of human cancer $(13,14)$. POSTN is able to interact with other ECM proteins, specific cell surface receptors and integrins through multiple signal pathways affecting metastasis, invasion and angiogenesis in cancer development $(15,16)$. Previous experimental results have revealed that POSTN has a role in mediating the promotion of esophageal cancer $(16,17)$. However, reports have yet to elucidate the association between POSTN and EMT in esophageal squamous cell carcinoma (ESCC). The present study hypothesized that POSTN is involved in the EMT of ESCC cells, and consequently facilitates cancer invasion and metastasis.

In the present study, the expression levels of POSTN, E-cad and Vim in ESCC cells and corresponding paracarcinomatous normal epithelium were examined using immunohistochemistry. The associations of POSTN, E-cad and Vim with clinicopathological features and the patients' survival status were investigated using univariate and multivariate analyses to verify whether POSTN is useful for predicting tumor malignant properties and poor prognosis in patients with ESCC.

\section{Materials and methods}

Patients and tumor biopsies. A total of 58 tumor specimens were obtained from patients with ESCC who underwent curative esophagectomy at the Department of Thoracic Surgery (Anhui Medical University, Hefei, China), between January 2007 and January 2008. All cases were diagnosed on a clinical basis with pathological confirmation and no patient received additional treatment prior to surgery. Of the 58 patients, there were 50 males and 8 females, with a median age of 61 years (range, 40-82). The obtained patient characteristics included tumor node metastasis (TNM) stage I/II $(n=16)$, TNM stage III/IV $(n=42)$, poor differentiation $(n=33)$, moderate and well differentiation $(n=25)$, lymphatic metastasis $(n=42)$ and vascular invasion $(n=44)$. Clinical status was determined in accordance with the National Comprehensive Cancer Network Clinical Practice Guidelines in Oncology Version 2009 (18). All patients' tissue specimens were fixed in $10 \%$ formalin, paraffin-embedded and stained with hematoxylin and eosin for immunohistochemical analysis. Clinical and pathological data of all patients was obtained from medical records, and the follow-up deadline was 17th March, 2015. The present study was approved by the Institutional Review Board of the Anhui Provincial Hospital (Anhui Medical University), and written informed consent was obtained from all participants.

Immunohistochemistry and evaluation. Immunohistochemical staining of POSTN, E-cad, and Vim in ESCC and adjacent normal tissues was performed by the two-step method (Beijing Zhongshan Jinqiao Biotechnology Co., Ltd., Beijing, China). Specimen sections (thickness, $4 \mu \mathrm{m}$ ) were dewaxed by dimethylbenzene, rinsed in phosphate-buffered saline (PBS), boiled under high pressure in citrate-buffer $(10 \mathrm{mmol} / \mathrm{l}$; $\mathrm{pH}=6.0)$ for 2 min for antigen retrieval, and treated with $3 \%$ hydrogen peroxide to block endogenous peroxidase activity. Each section was incubated with the primary mouse anti-E-cad monoclonal antibody (cat. no. ZM-0092; 1:200), mouse anti-Vim monoclonal antibody (cat. no. ZM-0260) (both 1:200; Beijing Zhongshan Jinqiao Biotechnology Co., Ltd.), and mouse anti-POSTN polyclonal antibody (cat. no. TA804575; 1:150; Abcam, Cambridge, UK) at $4{ }^{\circ} \mathrm{C}$ overnight. Subsequent to washing with PBS, the sections were incubated with the appropriate HRP-labeled secondary antibody (goat antimouse IgG; pre-diluted; from PV-6000 Polink-1 HRP DAB Detection System One-step polymer detection system for Mouse and Rabbit antibodies, Beijing Zhongshan Jinqiao Biotechnology Co., Ltd.) at room temperature for $30 \mathrm{~min}$. The reaction products were developed using a 3,3-diaminobenzidine (DAB; Beijing Zhongshan Jinqiao Biotechnology Co., Ltd.) solution for $10 \mathrm{~min}$, and dehydrated following hematoxylin counterstaining. Negative control slides were processed with PBS and a confirmed positive tissue section was used as a positive control.

Semi-quantitative integration was used to assess the results of immunohistochemistry. Yellow-brown granules located in cytoplasm and cytoplasmic membrane were defined as positive staining. Staining intensity was scored as follows: 0, negative; 1, yellow; 2, brown; and 3, sepia. An image of the background was taken for reference. Scores for the average percentage of immunopositive cells were based on the number of stained cells per 100 cells in 10 high-power fields (magnification, $\mathrm{x} 400$ ) from the hot spots in low power fields (magnification, $\mathrm{x} 100$ ), and rated as follows: $0,<10 \%$ positive cells; $1,10-25 \%$; $2,>25-50 \% ; 3,>50-75 \%$; and $4,>75 \%$ positive cells. The final assessment based on their product was as follows: 0 , negative $(-)$; $1-4$, weak positive $(+)$; 5-8, moderate positive $(++)$; and $9-12$, strong positive $(+++)$. The slices were independently evaluated by two pathologists who were blind to the clinical diagnosis.

Statistical analysis. Statistical analyses were completed with SPSS 13.0 (SPSS, Inc., Chicago, IL, USA). Experiments were performed in triplicate. All data were presented as the mean \pm standard deviation. The $\chi^{2}$ test was used as appropriate for the comparison of variables. Correlations between POSTN and EMT markers were performed using the Spearman's rank correlation test. Overall survival (OS) and disease-free survival (DFS) rates were determined using a Kaplan-Meier analysis and a log-rank test. The Cox regression model was used for multivariate survival analysis. $\mathrm{P}<0.05$ was considered to indicate a statistically significant difference.

\section{Results}

Expression levels of E-cad, Vim, and POSTN in the intratumoral and paracarcinomatous tissues of ESCC. E-cad was observed to be expressed on the squamous cell membranes, and attenuated notably in the ESCC tissues compared with the paracarcinomatous epithelium (32.8 vs. $74.1 \% ; \chi^{2}=19.957$; $\mathrm{P}<0.001$; Fig. 1). Vim was located in the cytoplasm of fibroblasts, endothelial cells and squamous cells; the focus of the present study was just squamous cells. Vim expression was positive in $24 / 58$ intratumoral cases $\left(41.4 \%, \chi^{2}=26.973, \mathrm{P}<0.001\right)$, significantly more than $1 / 58$ paracarcinomatous cases $(1.7 \%$; 
Table I. Positive rate of E-cad, Vim, and POSTN expression in the squamous cells of tumor and paracarcinomatous tissues in esophageal squamous cell carcinoma.

\begin{tabular}{lccccr}
\hline & \multicolumn{4}{c}{$\begin{array}{c}\text { Paracarcinomatous } \\
\text { tissues }\end{array}$} \\
\cline { 2 - 3 } Variable & $\begin{array}{c}\text { Positive } \\
\text { rate }(\%)\end{array}$ & & $\begin{array}{c}\text { Positive } \\
\text { rate }(\%)\end{array}$ & $\chi^{2}$ & P-value \\
\hline E-cad & $19 / 58(32.8)$ & $43 / 58(74.1)$ & 19.957 & $<0.001$ \\
Vim & $24 / 58(41.4)$ & $1 / 58(1.7)$ & 26.973 & $<0.001$ \\
POSTN & $41 / 58(70.7)$ & $23 / 58(39.7)$ & 11.293 & 0.001 \\
\hline
\end{tabular}

E-cad, epithelial cadherin; Vim, vimentin; POSTN, periostin.

Table II. Associations between POSTN and the EMT hallmark proteins in the esophageal squamous cell carcinoma squamous cells.

\begin{tabular}{ccccccc}
\hline & \multicolumn{2}{c}{ E-cad (SC) } & & \multicolumn{2}{c}{$\operatorname{Vim}(\mathrm{SC})$} \\
\cline { 2 - 3 } POSTN (SC) & r-value & P-value & & r-value & P-value \\
\hline & -0.339 & $<0.001$ & & 0.130 & $<0.001$
\end{tabular}

POSTN, periostin; EMT, epithelial-mesenchymal transition; E-cad, E-cadherin; Vim, vimentin; SC, squamous cell.

Fig. 1). POSTN expression was detected in the cytoplasm of fibroblasts, endothelial cells, and squamous cells. Similar to Vim, the present study also analyzed POSTN expression in squamous cells. The positive rate of POSTN expressed in the tumor squamous cells was $41 / 58\left(70.7 \%, \chi^{2}=11.293, \mathrm{P}=0.001\right)$, notably higher than $23 / 58(39.7 \%)$ in the paracarcinomatous epithelium (Fig. 1). Data above are presented in Table I.

Correlations between POSTN and the EMT hallmark proteins in ESCC squamous cells. The present study analyzed the correlations between POSTN and the EMT hallmark proteins E-cad and Vim in the ESCC squamous cells according to the immunohistochemical score. There was an inverse correlation between expression levels of E-cad and POSTN ( $r=-0.339$; $\mathrm{P}<0.001$; Table II). No case expressing E-cad was observed in the samples that had a POSTN score $>6$. By contrast, Vim expression was found to have a positive correlation with POSTN expression $(r=0.130 ; \mathrm{P}<0.001$; Table II). Intensity of Vim expression was weak to moderate/high, and Vim was observed to be expressed positively in the presence of POSTN. This trend in our experiment revealed that POSTN overexpression was associated with E-cad attenuation as well as Vim enrichment (Fig. 2).

Associations between clinicopathological characteristics and expression levels of E-cad, Vim, and POSTN in the ESCC squamous cells. The associations between expression levels
A

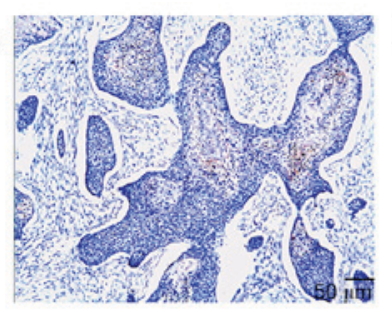

B

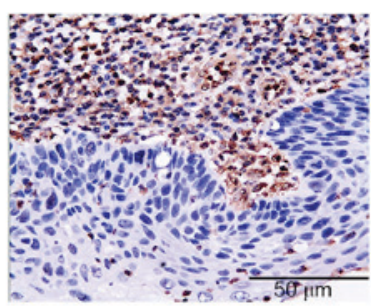

C
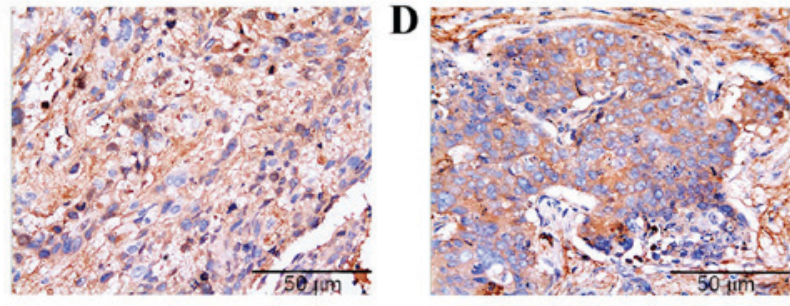

$\mathbf{E}$
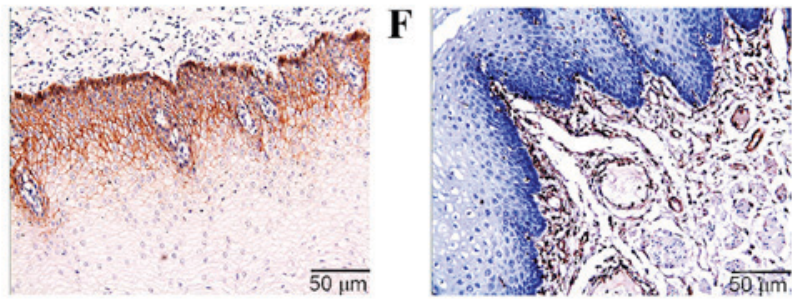

G

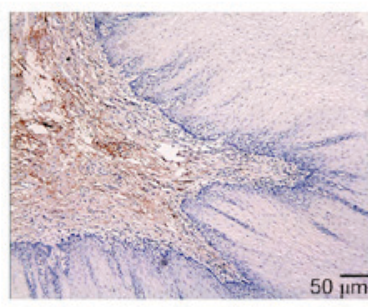

$\mathbf{H}$

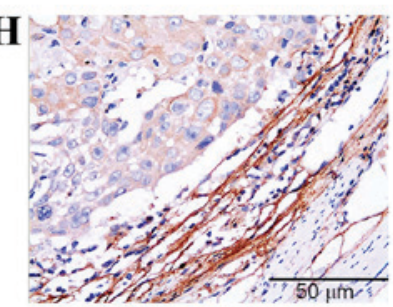

Figure 1. Immunohistochemistry demonstrates expression diversity of E-cad, Vim, and POSTN in the ESCC tissues. (A) E-cad expression in the tumor cells (magnification, $x 100$ ) receded significantly comparing with the strong staining in (E) the paracarcinomatous squamous cells (magnification, x200). (B) Vim expression in the tumor cells (magnification, x400) was stronger than that in $(\mathrm{F})$ the paracarcinomatous squamous cells (magnification, $\mathrm{x} 200$ ). (C) POSTN was observed to be overexpressed in the cancer cells (magnification, $\mathrm{x} 400$ ), whereas it demonstrated low positive rate in $(\mathrm{G})$ the paracarcinomatous squamous cells (magnification, x100). (D) POSTN expression in the tumor tissue with poor differentiation (magnification, $\mathrm{x} 400$ ) was denser than that in $(\mathrm{H})$ the well-differentiated tumor tissue (magnification, $\mathrm{x} 400$ ). Scale bar $=50 \mu \mathrm{m}$. E-cad, epithelial cadherin; Vim, vimentin; POSTN, periostin; ESCC, esophageal squamous cell carcinoma.

of the proteins in the ESCC squamous cells and clinicopathological factors were analyzed by the $\chi^{2}$ test. E-cad expression exhibited a higher positive rate in the tumor tissues with advanced TNM stage $\left(\chi^{2}=12.994 ; \mathrm{P}<0.001\right)$, lymphatic metastasis $\left(\chi^{2}=12.994 ; \mathrm{P}<0.001\right)$ and venous invasion $\left(\chi^{2}=4.981\right.$; $\mathrm{P}=0.026)$. Conversely, there was no statistical association between $\mathrm{E}$-cad status and tumor differentiation $(\mathrm{P}=0.647)$. The positive rate of Vim expression was elevated significantly in the cases with a terminal TNM stage $\left(\chi^{2}=4.665 ; \mathrm{P}=0.039\right)$ and lymphatic metastasis $\left(\chi^{2}=4.665 ; \mathrm{P}=0.039\right)$, but did not exhibit an association with the degree of tumor differentiation $(\mathrm{P}=0.072)$ or venous invasion $(\mathrm{P}=0.121)$. Notably, the overexpression of POSTN was validated to be significantly associated with all clinicopathological factors in this study, including TNM stage $\left(\chi^{2}=7.739 ; \mathrm{P}=0.009\right)$, tumor differentiation degree 

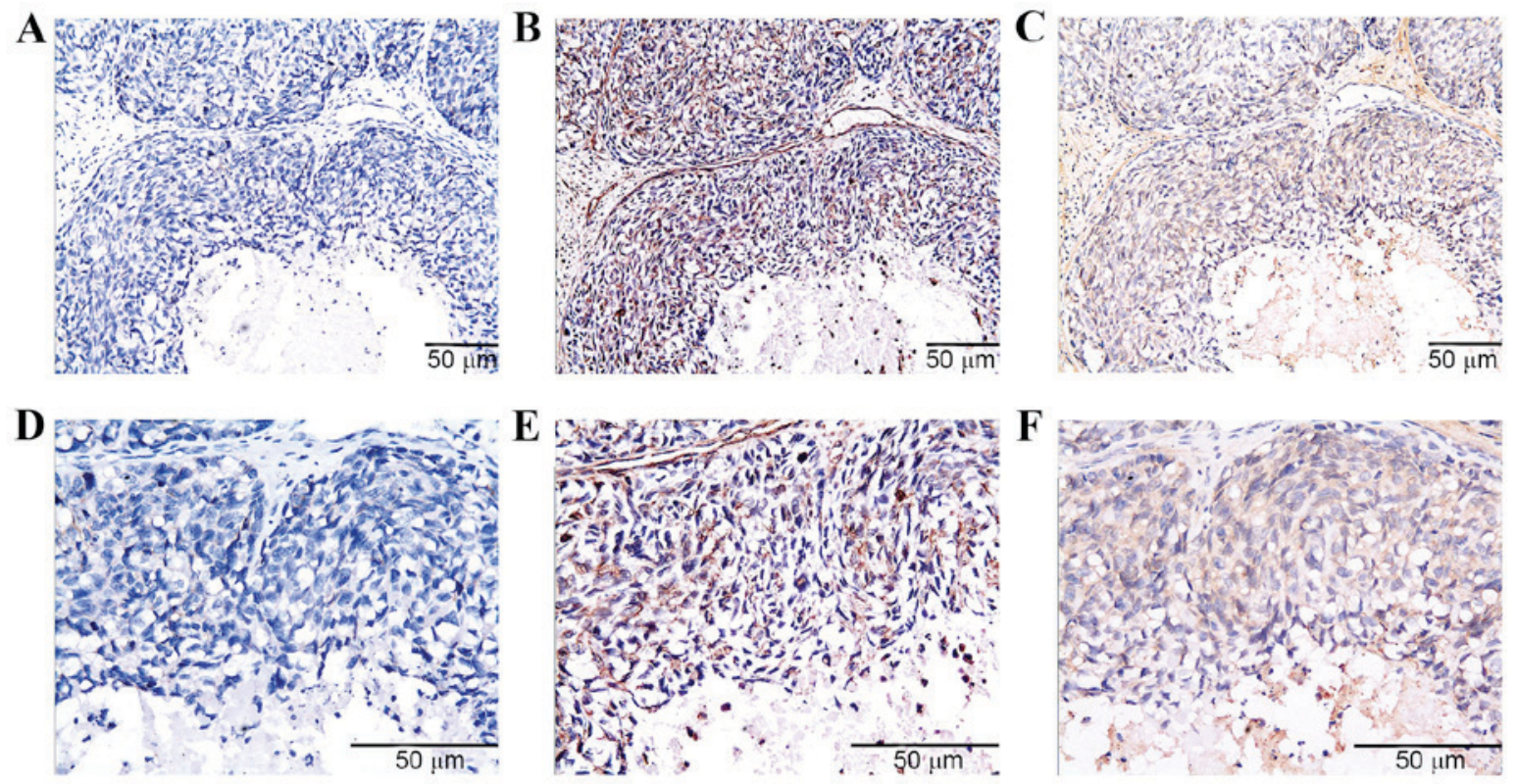

Figure 2. Consecutive slides from the same specimen of ESCC tissue exhibited the reciprocal association between the expression levels of POSTN and the EMT hallmark proteins. (A) Negative staining of E-cad in the cancer cells under low power lens (magnification, x200) and (D) high power lens (magnification, $\mathrm{x} 400$ ). (B) Positive staining of Vim in the cancer cells from the same location of the same specimen under low power lens (magnification, $\mathrm{x} 200$ ) and (E) high power lens (magnification, x400). (C) Positive staining of POSTN in the cancer cells from the same location of the same specimen under low power lens (magnification, x200) and (F) high power lens (magnification, x400). Scale bar=50 $\mu \mathrm{m}$. ESCC, esophageal squamous cell carcinoma; POSTN, periostin; EMT, epithelial-mesenchymal transition; E-cad, epithelial cadherin; Vim, vimentin.

$\left(\chi^{2}=15.106 ; \mathrm{P}<0.001 ;\right.$ Fig. 1$)$, lymphatic metastasis $\left(\chi^{2}=7.739\right.$; $\mathrm{P}=0.009)$ and venous invasion $\left(\chi^{2}=10.896 ; \mathrm{P}=0.002\right)$. These results are presented in detail in Table III.

Survival analysis. Survival analysis according to the Kaplan-Meier method revealed that the OS $(27.171 \pm 1.584$ months; Fig. 3A) and DFS (13.341 \pm 1.039 months; Fig. 3B) of patients with positive POSTN expression was markedly shorter than that of patients with negative POSTN expression (OS, 45.824 \pm 3.408 months, $\chi^{2}=22.252, \mathrm{P}<0.001$; DFS, $29.471 \pm 3.155$ months, $\left.\chi^{2}=22.696, \mathrm{P}<0.001\right)$. Similarly, patients with positive Vim expression tended to have a poorer prognosis for OS $\left(27.833 \pm 2.235\right.$ vs. $36.029 \pm 2.628$ months; $\chi^{2}=5.936$; $\mathrm{P}=0.015)$ and DFS (14.125 \pm 1.809 vs. $20.853 \pm 2.145$ months; $\left.\chi^{2}=5.750 ; \mathrm{P}=0.016\right)$. However, the OS $(40.895 \pm 3.327$ months $)$ and DFS (25.263 \pm 3.100 months) of patients with positive E-cad expression were markedly longer than those of patients with negative E-cad expression (OS, 28.615 \pm 1.968 months, $\chi^{2}=9.255$, $\mathrm{P}=0.002$; DFS, $14.564 \pm 1.389$ months, $\chi^{2}=9.667, \mathrm{P}=0.002$ ).

Multivariate analysis using the Cox regression model indicated that POSTN positive expression $(\mathrm{P}=0.022)$ and TNM stage $(\mathrm{P}=0.004)$ were independent prognostic factors for the OS of patients with ESCC. POSTN positive expression $(\mathrm{P}=0.019)$ remained one of the independent prognostic factors for the DFS of patients with ESCC, along with venous invasion $(\mathrm{P}=0.004)$ and TNM stage $(\mathrm{P}=0.008$; Table IV).

\section{Discussion}

EMT has been demonstrated to be involved in several underlying mechanisms leading to tumor invasion, migration and the induction of cancer stem-like cells in esophageal cancer $(19,20)$. In the present study, expression levels of E-cad and Vim in ESCC squamous cells changed abnormally and inversely. Reduction of the E-cad positive rate and elevation of the Vim positive rate in ESCC tissues indicated that EMT occurred in the ESCC tissues. In addition, attenuation of E-cad and enrichment of Vim in cancer cells was associated with the invasion and metastasis of ESCC, thus potentially affecting the prognosis of patients with ESCC.

The association between POSTN and EMT has been investigated in several types of tumors. However, conclusions vary depending on the type of cancer. POSTN is reported to be capable of enhancing EMT of nicotine-mediated tumor cells in lung and gastric cancer $(21,22)$, whereas exhibits biphasic effects on EMT in pancreatic cancer cells and even has a suppressive role in the regulation of EMT in bladder cancer cells $(23,24)$. The present study aimed to elucidate the reciprocal correlation between POSTN and EMT in ESCC, and revealed that POSTN overexpression was associated with E-cad attenuation as well as Vim enrichment, via immunohistochemistry.

Additionally, POSTN has been reported to be able to upregulate snail and twist, which are regarded as negative regulators of E-cad in transcription, and enhance protein kinase B (Akt) phosphorylation to decrease E-cad expression (24). POSTN was deemed to be involved in the crosstalk between epidermal growth factor receptor and integrins at the plasma membranes, activating the Akt and the focal adhesion kinase (FAK) signaling pathways consecutively to promote EMT in non-small cell lung, ovarian and colon cancer (25-27). Consequently, the present data showing the reciprocal correlations between POSTN and the EMT markers E-cad and Vim in ESCC are consistent with previous studies.

Considering the role of POSTN as a promotional mediator of EMT in ESCC cells, upregulated POSTN with concomitant repression of E-cad and overexpression of Vim may result in 
Table III. Associations between clinicopathological characteristics and positive rate of E-cad, Vim and POSTN in ESCC squamous cells.

\begin{tabular}{|c|c|c|c|c|c|c|c|c|c|c|}
\hline \multirow[b]{2}{*}{ Variable } & \multirow[b]{2}{*}{$\mathrm{n}$} & \multicolumn{3}{|c|}{ E-cad } & \multicolumn{3}{|c|}{ Vim } & \multicolumn{3}{|c|}{ POSTN } \\
\hline & & $\begin{array}{l}\text { Positive } \\
\text { rate, \% }\end{array}$ & $\chi^{2}$ & P-value & $\begin{array}{l}\text { Positive } \\
\text { rate, } \%\end{array}$ & $\chi^{2}$ & P-value & $\begin{array}{l}\text { Positive } \\
\text { rate, } \%\end{array}$ & $\chi^{2}$ & P-value \\
\hline \multicolumn{11}{|l|}{ TNM stage } \\
\hline $\mathrm{I} / \mathrm{II}$ & 16 & 68.8 & 12.994 & $<0.001$ & 18.8 & 4.665 & 0.039 & 43.8 & 7.739 & 0.009 \\
\hline III/IV & 42 & 19.0 & & & 50.0 & & & 81.0 & & \\
\hline \multicolumn{11}{|c|}{ Differentiation degree } \\
\hline Low & 33 & 30.3 & 0.210 & 0.647 & 51.5 & 3.243 & 0.072 & 90.9 & 15.106 & $<0.001$ \\
\hline Moderate/high & 25 & 36.0 & & & 28.0 & & & 44.0 & & \\
\hline \multicolumn{11}{|c|}{ Lymphatic metastasis } \\
\hline Positive & 42 & 19.0 & 12.994 & $<0.001$ & 50.0 & 4.665 & 0.039 & 81.0 & 7.739 & 0.009 \\
\hline Negative & 16 & 68.8 & & & 18.8 & & & 43.8 & & \\
\hline \multicolumn{11}{|l|}{ Venous invasion } \\
\hline Positive & 44 & 25.0 & 4.981 & 0.026 & 47.7 & 3.028 & 0.121 & 81.8 & 10.896 & 0.002 \\
\hline Negative & 14 & 57.1 & & & 21.4 & & & 35.7 & & \\
\hline
\end{tabular}

E-cad, epithelial cadherin; Vim, vimentin; POSTN, periostin; ESCC, esophageal squamous cell carcinoma.

A

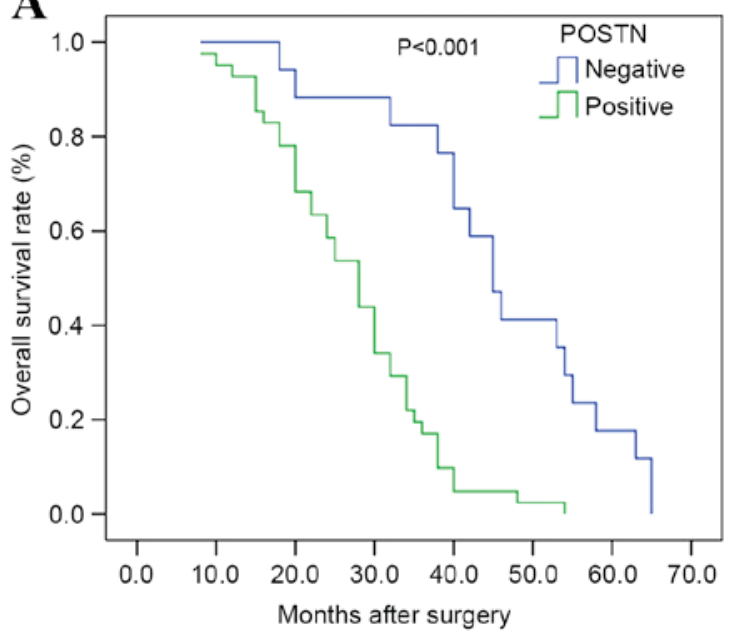

B

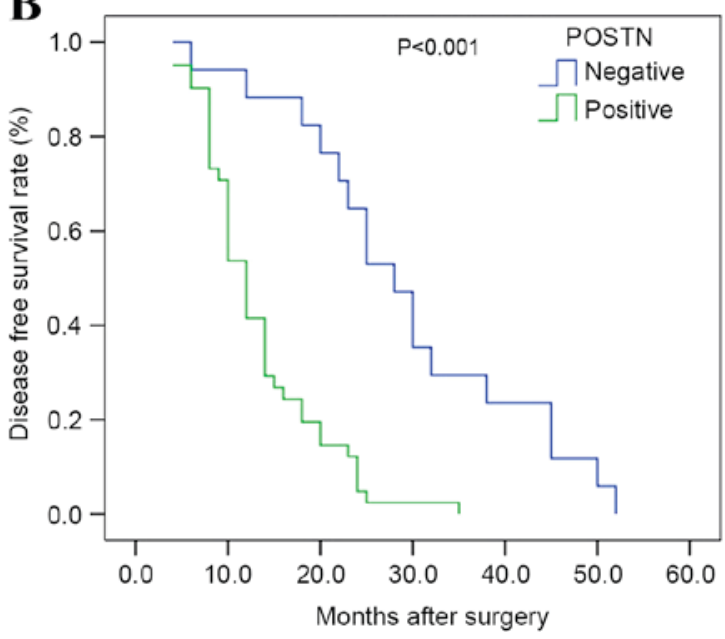

Figure 3. Kaplan-Meier analysis of OS rate DFS rate curves of patients with ESCC based on POSTN expression as positive or negative. The ESCC patients with POSTN positive expression had significantly shorter period than those with POSTN negative expression in (A) OS and (B) DFS. P-values were calculated using the log-rank test. OS, overall survival; DFS, disease free survival; ESCC, esophageal squamous cell carcinoma; POSTN, periostin.

advanced tumor characteristics. The present study therefore demonstrated that POSTN expression was elevated in the ESCC tissues with poor differentiation, lymphatic metastasis, venous invasion and thus a severe TNM stage. In terms of the mechanism, POSTN was previously reported to be able to combine to multiple integrin receptors including $\alpha v \beta 3$, $\alpha v \beta 5$, and $\alpha 6 \beta 4$, partially regulating the intracellular signaling pathways linked to phosphatidylinositol 3 kinase (PI3K) /AKT as well as FAK, and sequentially induce the proliferation, metastasis and invasion of cancer cells, and alter tumor microenvironment $(11,16,28)$. The results of the present study validate the negative influences on the ESCC tissue due to the increased POSTN expression in cancer cells. In addition, previous studies of POSTN in esophageal cancer accentuated the significance of microenvironment invasion mediated by POSTN, suggesting that POSTN may induce vascular endothelial growth factor expression and cooperate with mutant P53 to promote tumor angiogenesis and invasion in microenvironment $(16,29,30)$. These findings suggest that POSTN may be responsible for malignant clinicopathological properties, and could therefore be considered as a predictive marker for the presence of lymph node and blood vessel invasion as well as histologically poor differentiation.

Since the functions of POSTN lead to the aggressive properties in ESCC cells, the survival period could be affected in the patients with these features induced by upregulated POSTN expression. The OS and DFS of POSTN positive 
Table IV. Multivariate analysis of factors associated with OS and DFS in the ESCC patients.

\begin{tabular}{|c|c|c|c|c|c|c|}
\hline Factors & $\begin{array}{l}\text { OS } \\
\text { RR }\end{array}$ & RR 95\% CI & P-value & $\begin{array}{c}\text { DFS } \\
\text { RR }\end{array}$ & RR 95\% CI & P-value \\
\hline \multicolumn{7}{|l|}{ POSTN } \\
\hline Positive vs. negative & 3.126 & $1.177-8.300$ & 0.022 & 2.721 & $1.180-6.277$ & 0.019 \\
\hline \multicolumn{7}{|l|}{ E-cad } \\
\hline Positive vs. negative & 0.852 & $0.378-1.920$ & 0.699 & 0.687 & $0.305-1.547$ & 0.365 \\
\hline \multicolumn{7}{|l|}{ Vim } \\
\hline Positive vs. negative & 1.067 & $0.524-2.170$ & 0.859 & 1.122 & $0.569-2.212$ & 0.739 \\
\hline \multicolumn{7}{|l|}{ Gender } \\
\hline Male vs. female & 1.918 & $0.812-4.526$ & 0.137 & 2.085 & $0.877-4.957$ & 0.096 \\
\hline \multicolumn{7}{|l|}{ Age } \\
\hline$\geq 60$ vs. $<60$ & 1.345 & $0.590-3.066$ & 0.481 & 1.823 & $0.810-4.104$ & 0.147 \\
\hline \multicolumn{7}{|l|}{ TNM stage } \\
\hline III/IV vs. I/II & 4.600 & $1.649-12.834$ & 0.004 & 3.788 & $1.422-10.090$ & 0.008 \\
\hline \multicolumn{7}{|l|}{ Differentiation degree } \\
\hline Moderate/high vs. low & 0.581 & $0.255-1.320$ & 0.195 & 0.723 & $0.359-1.455$ & 0.363 \\
\hline \multicolumn{7}{|l|}{ Venous invasion } \\
\hline Positive vs. negative & 2.250 & $0.871-5.813$ & 0.094 & 4.174 & $1.567-11.118$ & 0.004 \\
\hline
\end{tabular}

OS, overall survival; DFS, disease free survival; ESCC, esophageal squamous cell carcinoma; RR, relative risk; CI, confidence interval; POSTN, periostin; E-cad, epithelial cadherin; Vim, vimentin; TNM, tumor node metastasis.

patients with ESCC were both markedly shorter than that of the POSTN negative patients in the univariate analysis. Additionally, multivariate analysis revealed that overexpression of POSTN in the ESCC cells can predict poor prognosis independently in OS and DFS. These data are in agreement with the negative impact of POSTN on the patients' survival in non-small cell lung cancer, breast, colorectal, prostate, and ovarian cancers (31-35). The present results therefore indicated the pivotal role of POSTN in the progression of invasion and metastasis in ESCC tissues, as well as the prognostic value in the survival of patients with ESCC.

There were several limitations to the present study. Immunohistochemistry could only reflect the correlations between POSTN and EMT. Therefore, transfection of target genes, cytological analyses and xenografts on animal models are required to further the understanding of these correlations. In addition, a bias may have occurred due to the insufficient sample amount. The above limitations made the present conclusions preliminary.

In conclusion, the present study revealed that POSTN could be regarded as an upregulated factor in ESCC cells, promoting EMT, facilitating the invasion and metastasis of tumor cells, thus leading to a poor prognosis. These results indicated that suppression of the regulation between POSTN and EMT may provide a valid means to control tumor metastasis and the relapse of patients with ESCC.

\section{Acknowledgements}

The authors would like to thank Dr Ke Chen and Dr Xiao-Qiu Wang (Department of Pathology, Anhui Provincial Hospital,
Hefei, China) for their assistance with pathology. The present study was partly supported by the National Natural Science Foundation of China (grant nos. 81472329 and 81201906).

\section{References}

1. Lin Y, Totsuka Y, He Y, Kikuchi S, Qiao Y, Ueda J, Wei W, Inoue $\mathrm{M}$ and Tanaka $\mathrm{H}$ : Epidemiology of esophageal cancer in Japan and China. J Epidemiol 23: 233-242, 2013.

2. Tu CC, Hsu PK, Chien LI, Liu WC, Huang CS, Hsieh CC, Hsu HS and Wu YC: Prognostic histological factors in patients with esophageal squamous cell carcinoma after preoperative chemoradiation followed by surgery. BMC Cancer 17: 62, 2017.

3. Marcucci F, Stassi G and De Maria R: Epithelial-mesenchymal transition: A new target in anticancer drug discovery. Nat Rev Drug Discov 15: 311-325, 2016.

4. Miyoshi A, Kitajima Y, Sumi K, Sato K, Hagiwara A, Koga Y and Miyazaki K: Snail and SIP1 increase cancer invasion by upregulating MMP family in hepatocellular carcinoma cells. Br J Cancer 90: 1265-1273, 2014.

5. Jang SY, Park SY, Lee HW, Choi YK, Park KG, Yoon GS, Tak WY, Kweon YO, Hur K and Lee WK: The combination of periostin overexpression and microvascular invasion is related to a poor prognosis for hepatocellular carcinoma. Gut Liver 10: 948-954, 2016.

6. Vardaki I, Ceder S, Rutishauser D, Baltatzis G, Foukakis T and Panaretakis T: Periostin is identified as a putative metastatic marker in breast cancer-derived exosomes. Oncotarget 7: 74966-74978, 2016.

7. Molena D, Mungo B, Stem M, Poupore AK, Chen SY and Lidor AO: Does quality of care matter? A study of adherence to national comprehensive cancer network guidelines for patients with locally advanced esophageal cancer. J Gastrointest Surg 19: 1739-1747, 2015.

8. Jiang Y, Liao L, Shrestha C, Ji S, Chen Y, Peng J, Wang L, Liao E and Xie Z: Reduced expression of E-cadherin and p120-catenin and elevated expression of PLC- $\gamma 1$ and PIKE are associated with aggressiveness of oral squamous cell carcinoma. Int J Clin Exp Pathol 8: 9042-9051, 2015. 
9. Lanier MH, Kim T and Cooper JA: CARMIL2 is a novel molecular connection between vimentin and actin essential for cell migration and invadopodia formation. Mol Biol Cell 26 4577-4588, 2015

10. Paccione RJ, Miyazaki H, Patel V, Waseem A, Gutkind JS, Zehner ZE and Yeudall WA: Keratin down-regulation in vimentin-positive cancer cells is reversible by vimentin RNA interference, which inhibits growth and motility. Mol Cancer Ther 7: 2894-2903, 2008.

11. Bayo P, Jou A, Stenzinger A, Shao C, Gross M, Jensen A Grabe N, Mende CH, Rados PV, Debus J, et al: Loss of SOX2 expression induces cell motility via vimentin up-regulation and is an unfavorable risk factor for survival of head and neck squamous cell carcinoma. Mol Oncol 9: 1704-1719, 2015.

12. Jin MS, Hyun CL, Park IA, Kim JY, Chung YR, Im SA, Lee KH, Moon HG and Ryu HS: SIRT1 induces tumor invasion by targeting epithelial mesenchymal transition-related pathway and is a prognostic marker in triple negative breast cancer. Tumour Biol 37: 4743-4753, 2016

13. Yan TT, Fu XL, Li J, Bian YN, Liu DJ, Hua R, Ren LL, Li CT, Sun YW, Chen HY, et al: Downregulation of RPL15 may predict poor survival and associate with tumor progression in pancreatic ductal adenocarcinoma. Oncotarget 6: 37028-37042, 2015.

14. Hu Q, Tong S, Zhao X, Ding W, Gou Y, Xu K, Sun C and Xia G: Periostin mediates TGF- $\beta$-induced epithelial mesenchymal transition in prostate cancer cells. Cell Physiol Biochem 36: 799-809, 2015.

15. Litvin J, Selim AH, Montgomery MO, Lehmann K, Rico MC, Devlin H, Bednarik DP and Safadi FF: Expression and function of periostin-isoforms in bone. J Cell Biochem 92: 1044-1061, 2004.

16. Contié S, Voorzanger-Rousselot N, Litvin J, Clézardin P and Garnero P: Increased expression and serum levels of the stromal cell-secreted protein periostin in breast cancer bone metastases. Int J Cancer 128: 352-360, 2011

17. Wang W, Sun QK, He YF, Ma DC, Xie MR, Ji CS and Hu B: Overexpression of periostin is significantly correlated to the tumor angiogenesis and poor prognosis in patients with esophageal squamous cell carcinoma. Int J Clin Exp Pathol 7: 593-601, 2014.

18. Underwood TJ, Hayden AL, Derouet M, Garcia E, Noble F, White MJ, Thirdborough S, Mead A, Clemons N, Mellone M, et al: Cancer-associated fibroblasts predict poor outcome and promote periostin-dependent invasion in oesophageal adenocarcinoma. J Pathol 235: 466-477, 2015.

19. Li S, Qin X, Li Y, Zhang X, Niu R, Zhang H, Cui A, An W and Wang X: MiR-133a suppresses the migration and invasion of esophageal cancer cells by targeting the EMT regulator SOX4. Am J Transl Res 7: 1390-1403, 2015.

20. Sato F, Kubota Y, Natsuizaka M, Maehara O, Hatanaka Y, Marukawa K, Terashita K, Suda G, Ohnishi S, Shimizu Y, et al: EGFR inhibitors prevent induction of cancer stem-like cells in esophageal squamous cell carcinoma by suppressing epithelial-mesenchymal transition. Cancer Biol Ther 16: 933-940, 2015

21. Wu SQ, Lv YE, Lin BH, Luo LM, Lv SL, Bi AH and Jia YS Silencing of periostin inhibits nicotine-mediated tumor cell growth and epithelial-mesenchymal transition in lung cancer cells. Mol Med Rep 7: 875-880, 2013.
22. Liu Y and Liu BA: Enhanced proliferation, invasion, and epithelial-mesenchymal transition of nicotine-promoted gastric cancer by periostin. World J Gastroenterol 17: 2674-2680, 2011.

23. Kanno A, Satoh K, Masamune A, Hirota M, Kimura K, Umino J, Hamada S, Satoh A, Egawa S, Motoi F, et al: Periostin, secreted from stromal cells, has biphasic effect on cell migration and correlates with the epithelial to mesenchymal transition of human pancreatic cancer cells. Int J Cancer 122: 2707-2718, 2008.

24. Kim CJ, Sakamoto K, Tambe Y and Inoue H: Opposite regulation of epithelial-to-mesenchymal transition and cell invasiveness by periostin between prostate and bladder cancer cells. Int J Oncol 38: 1759-1766, 2011.

25. Soltermann A, Tischler V, Arbogast S, Braun J, Probst-Hensch N, Weder W, Moch H and Kristiansen G: Prognostic significance of epithelial-mesenchymal and mesenchymal-epithelial transition protein expression in non-small cell lung cancer. Clin Cancer Res 14: 7430-7437, 2008.

26. Gillan L, Matei D, Fishman DA, Gerbin CS, Karlan BY and Chang DD: Periostin secreted by epithelial ovarian carcinoma is a ligand for alpha (V)beta(3) and alpha(V)beta(5) integrins and promotes cell motility. Cancer Res 62: 5358-5364, 2002.

27. Bao S, Ouyang G, Bai X, Huang Z, Ma C, Liu M, Shao R, Anderson RM, Rich JN and Wang XF: Periostin potently promotes metastatic growth of colon cancer by augmenting cell survival via the Akt/PKB pathway. Cancer Cell 5: 329-339, 2004.

28. Mosher DF, Johansson MW, Gillis ME and Annis DS: Periostin and TGF- $\beta$-induced protein: Two peas in a pod? Crit Rev Biochem Mol Biol 50: 427-439, 2015.

29. Heidari P, Esfahani SA, Turker NS, Wong G, Wang TC, Rustgi AK and Mahmood U: Imaging of secreted extracellular periostin, an important marker of invasion in the tumor microenvironment in esophageal cancer. J Nucl Med 56: 1246-1251, 2015.

30. Wong GS, Lee JS, Park YY, Klein-Szanto AJ, Waldron TJ, Cukierman E, Herlyn M, Gimotty P, Nakagawa H and Rustgi AK: Periostin cooperates with mutant p53 to mediate invasion through the induction of STAT1 signaling in the esophageal tumor microenvironment. Oncogenesis 2: e59, 2013.

31. Hong LZ, Wei XW, Chen JF and Shi Y: Overexpression of periostin predicts poor prognosis in non-small cell lung cancer. Oncol Lett 6: 1595-1603, 2013

32. Nuzzo PV, Rubagotti A, Argellati F, Di Meglio A, Zanardi E, Zinoli L, Comite P, Mussap M and Boccardo F: Prognostic value of preoperative serum levels of periostin (PN) in early breast cancer (BCa). Int J Mol Sci 16: 17181-17192, 2015.

33. Li Z, Zhang X, Yang Y, Yang S, Dong Z, Du L, Wang L and Wang C: Periostin expression and its prognostic value for colorectal cancer. Int J Mol Sci 16: 12108-12118, 2015.

34. Nuzzo PV, Rubagotti A, Zinoli L, Ricci F, Salvi S, Boccardo S and Boccardo F: Prognostic value of stromal and epithelial periostin expression in human prostate cancer: Correlation with clinical pathological features and the risk of biochemical relapse or death. BMC Cancer 12: 625, 2012.

35. Ryner L, Guan Y, Firestein R, Xiao Y, Choi Y, Rabe C, Lu S, Fuentes E, Huw LY, Lackner MR, et al: Upregulation of periostin and reactive stroma is associated with primary chemoresistance and predicts clinical outcomes in epithelial ovarian cancer. Clin Cancer Res 21: 2941-2951, 2015. 